\title{
Enjeux et opportunités de l'entreprise réseau
}

\author{
Afef El Herelli \\ École Supérieure de Commerce de Tunis
}

Garantir la meilleure performance dans toutes ses activités est l'un des principaux objectifs de l'entreprise. Mais cette dernière, et notamment la PME, ne peut pas exceller dans toutes ses activités. La nécessité de s'associer à d'autres acteurs afin de profiter de la complémentarité des ressources et des compétences, partager les risques et faire face aux aléas de l'environnement devient urgente.

Fonctionner ainsi dans le cadre d'un réseau vertical associant des entreprises complémentaires offre plusieurs opportunités mais également pose plusieurs enjeux. L'objet de cet article est d'apporter une meilleure compréhension à la logique de l'entreprise réseau à travers la justification du passage vers ce mode de coopération interentreprises, la présentation des diverses opportunités et potentialités offertes aux entreprises appartenant au réseau ainsi que les enjeux posés qui, non pris en considération par l'entreprise, peuvent représenter des limites ou des entraves face à la réussite de l'entreprise réseau.

\section{Les fondements de l'entreprise réseau :}

L'entreprise réseau représente une forme de collaboration interentreprises et a pour vocation de répondre aux exigences nées des nouvelles contraintes environnementales imposées. Elle se constitue lorsque les opérations productives ont une dimension verticale autour d'une entreprise pivot, qui voit graviter autour d'elle des opérateurs spécialisés selon leurs compétences distinctives. Les entreprises faisant partie du réseau sont juridiquement et financièrement indépendantes et mettent en commun leurs ressources et leurs compétences grâce à des relations contractuelles. $L$ 'entreprise pivot se focalise sur son cœur de métier et confie certaines de ses activités à d'autres organisations capables de les réaliser à moindre réaliser à moindre coût. Elle est également qualifiée de strategic center, hub firm, ou de broker. Toyota, par exemple, est composée de fournisseurs indépendants, de producteurs choisis en fonction de leur compétitivité et de leur degré de loyauté à l'égard du pivot.

L'entreprise réseau est basée sur le recentrage sur le cœur de métier et la coopération verticale. Le recentrage implique une concentration des ressources disponibles sur les activités rentables et dans lesquelles l'entreprise peut jouer un rôle dominant sur le marché. Le métier de base se définit ainsi par les ressources et les compétences mobilisées pour obtenir les produits plutôt que par les produits eux-mêmes ou le portefeuille d'activités.

La focalisation sur le cœur de métier se traduit par l'externalisation d'activités non prioritaires ou estimées peu performantes. L'externalisation consiste dans le recours à un prestataire ou à un fournisseur externe pour une activité qui était jusqu'alors réalisée au sein de l'entreprise. Elle s'accompagne fréquemment d'un transfert de ressources matérielles ou humaines vers le prestataire choisi. L'externalisation peut commencer par les activités loin du cœur de métier et le passage vers le travail en réseau s'effectue de manière progressive jusqu'à toucher les activités proches du cœur de métier.

La coopération verticale interentreprises représente un autre fondement de l'entreprise pivot. Chaque entreprise intervient dans son domaine d'expertise ou son corps de métier. En effet, la recherche continue davantage concurrentielle. La garantie de pérennité ne peut plus s'effectuer de façon indépendante. L'entreprise sera incitée à agencer avec d'autres opérateurs ses activités et ses 
tres opérateurs ses activités et ses ressources selon une logique de processus.

L'émergence de l'entreprise réseau est justifiée par plusieurs facteurs. Nous présenterons dans le cadre du paragraphe suivant les conditions qui ont favorisé son adoption comme mode d'organisation pouvant présenter une solution face aux contraintes environnementales.

\section{Les conditions d'émergence de l'entreprise réseau : mutations organisationnelles et économiques}

L'adoption de ce modèle d'organisation interentreprises a été justifiée à la fois théoriquement qu'empiriquement. Le réseau représente un moyen efficace pour gérer la complexité grâce à la spécialisation et à la répartition des rôles entre les entreprises intervenant dans le réseau. En effet, l'émergence des entreprises réseau est liée à plusieurs facteurs, citons notamment les changements perpétuels de l'environnement qui ont permis de noter les limites des deux modèles économiques de référence que sont le marché et la hiérarchie et, par conséquent, la tendance vers le recentrage. Plusieurs auteurs expliquent le développement de l'entreprise réseau par les avancées réalisées dans le domaine des technologies de l'information et de la communication.

\section{Les facteurs environnementaux : explication par les grands événements dans l'environnement de l'entreprise}

L'environnement international de l'entreprise a évolué durant les phases de l'histoire. En effet, les grandes récessions ont souvent réduit les échanges internationaux. Mais après les années de guerre, l'industrie a dû se reconstruire et inventer de nouveaux modèles d'organisation. Les trente glorieuses (1945-1973) ont été le résultat des nouvelles formes productions et des échanges internationaux, basés sur la standardisation et la production de masse.

Vers la fin des années 1960, une prise de conscience de la part des employés, qui ont constaté que le travail était répétitif, et des consommateurs, qui sont devenus exigeants, a permis de passer d'une économie d'offre à une économie de demande. Ce changement a amené les grandes entreprises à délaisser leur système de production rigide et intégré, centré sur la quantité, et qui offrait un minimum de service. À ce système s'est substitué un nouveau mode de production flexible proposant de plus en plus de qualité et se renouvelant très souvent, qui s'adresse à des clients critiques et ayant des goûts disparates. Les entreprises cherchent ainsi de nouvelles façons de faire en passant vers de nouvelles formes décentralisées d'organisations innovantes et développant des relations de coopération avec d'autres entreprises.

L'efficacité des relations partenariales et du développement de réseaux a été prouvée dans le contexte japonais. Le toyotisme basé sur une nouvelle forme de production en réseau a remplacé le système de production concentré. Ce mode d'organisation a permis une amélioration de la qualité des produits et une facilité à intégrer l'innovation.

On peut résumer la pensée de M. Toyota en rappelant qu'une entreprise ne peut tout faire seule ou, en tout cas, tout bien faire. Il convient donc qu'elle concentre sa production là où elle a les plus grands avantages. Dans le cas de la firme Toyota, on s'est concentré sur la conception des voitures et leur montage ou assemblage, tout en travaillant avec les meilleurs producteurs de pièces, et en profitant de leurs savoirs et de leurs capacités de développement.

Julien et al. $\left(2003^{1}\right) P 9$.

Pour Julien et al. (2003), le défi de l'innovation ne peut être réalisé que collectivement et, en particulier, grâce aux réseaux. Le passage vers l'entreprise réseau vise ainsi à partager non seulement les risques du changement mais aussi et surtout le savoir et les capacités d'apprentissage. Le réseautage ainsi créé favorise l'amélioration et la transformation continue de la production et engendre systématiquement de l'innovation.

Le passage vers le travail en réseau montra alors son efficacité en termes de qualité, d'innovation et d'augmentation de parts de marchés et montra ainsi les limites des deux modèles économiques de référence que sont le marché et la hiérarchie. 


\section{Explication économique : limites des modèles de référence}

Figure 1 : Formes hybrides et fréquence de perturbation selon Williamson

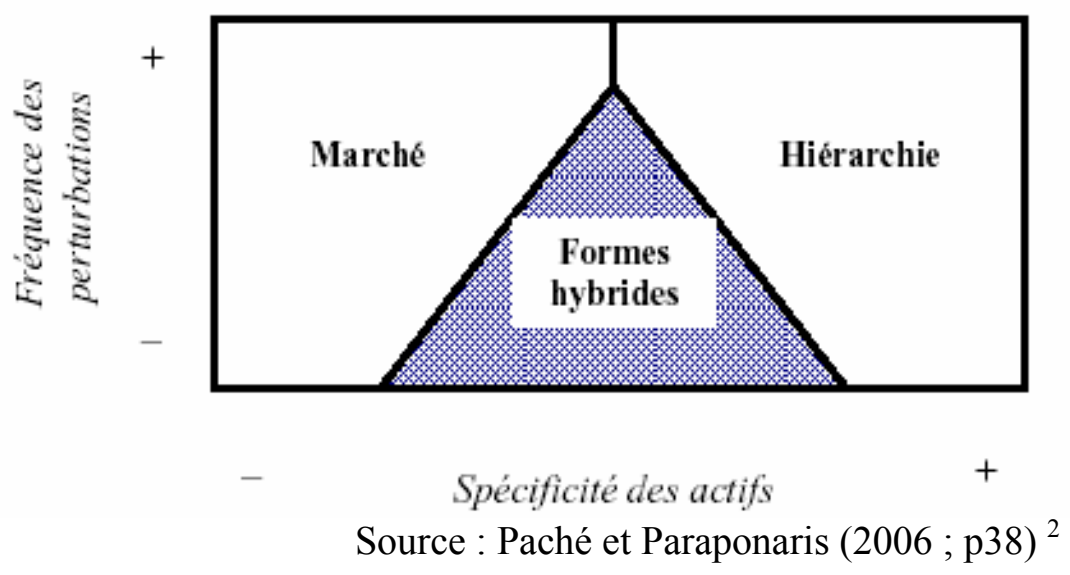

D'un point de vue économique, il est désormais reconnu que les insuffisances des deux modèles opposés (le marché et la hiérarchie) sont à l'origine de l'émergence des entreprises réseau. Les modèles économiques de référence que sont le marché et la hiérarchie ont fait l'objet de nombreuses recherches.

Le modèle de marché se caractérise par le principe d'allocation optimale des ressources ainsi que celui de coordination des acteurs sur le marché par l'intermédiaire des prix.

Le modèle intégrateur basé sur la stratégie d'intégration se révèle approprié en termes d'économies d'échelle, de contrôle des approvisionnements, d'économies induites par les innovations des processus ou de produits et de barrières à l'entrée (effet de taille). Ce modèle s'est imposé jusqu'au milieu des années 1970.

Les conditions environnementales (développées dans le paragraphe précédent) ont déclenché un mouvement inverse de désintégration verticale. La tendance au recentrage des grandes entreprises sur leur métier de base, amorcée au début des années 1980, les a conduites à se restructurer. De nouvelles relations, correspondant à des démarches de coopération, tendent à transcender la simple alternative marché/hiérarchie. Ces relations se fondent simultanément sur ces deux modèles en tentant de cumuler leurs avantages et d'éviter leurs inconvénients respectifs (les comportements opportunistes à court terme et un champ de vision étroit, pour le modèle marchand, et un coût élevé de gestion de l'information et une certaine rigidité des comportements pour le modèle intégrateur).

De plus, Gianfoldoni et al. (1997) ${ }^{3}$ avancent que « la crise de modèle intégrateur peut être perçue comme découlant d'une trop grande rigidité structurelle, et la crise du modèle marchand trouve ses origines dans l'instabilité structurelle qu'il prédispose (opportunismes comportementaux et incertitudes relationnelles) ».

Dans ce même cadre, Gianfoldoni et al. (1997) ont analysé les entreprises en réseau dans l'industrie du Bâtiment et ont souligné les insuffisances des deux modèles traditionnels en explicitant les raisons de la désintégration verticale des entreprises dans cette industrie. Trois types de raisons ont été notés à savoir :

- commerciales : diminution des marchés potentiels, baisse des marges anticipées liée à une concurrence accrue, hausse des exigences des clients ;

- productives : "recentrage de l'entreprise générale sur les activités d'études de marchés, de conception et de gestion de projets, planification ex ante de la production et des marges bénéficiaires, délégation d'une part croissante de la production réelle et des prises de risque d'investissements en équipements productifs, ̀̀ des $P M E »$;

- financières : «repositionnement de l'entreprise générale sur des nouvelles formes d'investissement à rentabiliser (conception et 
maîtrise technologique de nouveaux systèmes d'information) et stratégie de captation des profits « sûrs » de la part du holding »

Le nouveau modèle d'organisation se situe ainsi entre les deux modèles polaires traditionnels. De nouvelles relations interentreprises concrétisées par la mise en œuvre de stratégies de coopération donnent naissance à un nouveau modèle d'organisation situé entre les deux modèles polaires traditionnels. Les entreprises en réseau se rapprochent simultanément des relations marchandes (indépendance des échangistes) et des relations hiérarchiques (mode de gestion fondé sur la collaboration).

Les raisons économiques à l'origine de l'émergence des structures en réseau se situent ainsi à trois niveaux : les défaillances du modèle marchand, les défaillances du modèle intégrateur et le processus stratégique de recentrage des grandes entreprises.

\section{Les conditions économiques, technologi- ques et organisationnelles sont à l'origine de l'émergence de l'entreprise réseau.}

\section{Le développement des TIC et le passage à l'entreprise virtuelle}

Il est évident que l'utilisation des technologies de l'information dans les entreprises réseau permet de faciliter la communication et l'échange de l'information. Certains auteurs associent le développement et l'émergence des entreprises réseau à l'utilisation des nouvelles technologies qui ont facilité remarquablement la collaboration interentreprises et notamment grâce aux systèmes EDI (Electronic Data Interchange).

Fréry (2001) ${ }^{4}$ s'est intéressé à un cas particulier d'entreprise réseau basée sur les technologies de l'information: l'entreprise virtuelle. Il considère que les technologies de l'information ont permis de remplacer l'intégration verticale capitalistique par une intégration électronique (Guilloux, 1994) pour arriver à une entreprise virtuelle que l'on peut définir comme un réseau de firmes indépendantes mettant en commun leurs ressources et compétences grâce aux technologies de l'information en des si- tuations de cotraitance; chacune étant spécialisée sur une étape de la chaîne de valeur à construire.

Pour Paché et Bacus-montfort (2002) ${ }^{5}$, « La firme à l'initiative $d u$ réseau opte pour une croissance contractuelle qui mobilise des actifs plus ou moins spécifiques auprès de divers partenaires, plutôt que d'en avoir la propriété. Cette forme dont l'existence est liée au développement des NTIC facilite la gestion des interdépendances et procure une grande flexibilité, mais au prix d'une évolution des rôles et compétences des acteurs.»

Les conditions économiques, technologiques et organisationnelles sont à l'origine de l'émergence de l'entreprise réseau. Cette dernière présente des atouts et des potentialités qui feront l'objet du paragraphe suivant.

\section{Les atouts de l'entreprise réseau}

Plusieurs auteurs se sont intéressés aux avantages des entreprises réseau. Cependant, il existe un ensemble de conditions que nous considérons comme des préalables à la réalisation des avantages à savoir :

- la confiance réciproque basée sur la transparence

- proximité géographique ou sociopsychologique favorisant la communication et l'échange d'informations

- la logique de concurrence coopération : La logique de concurrence se concrétise par le fait que l'entreprise pivot ou le donneur d'ordres peut choisir plus qu'un fournisseur pour un produit déterminé. De même, le preneur d'ordres cherche à limiter sa dépendance vis-à-vis du pivot en opérant avec d'autres donneurs d'ordres ou en développant des produits maison.

- les contrats à long terme basé sur la confiance

- un même niveau technologique

- les moyens de réalisation des objectifs établis permettant d'effectuer l'activité objet de coopération

Les principaux avantages réalisés suite à la vérification de ces conditions sont : la maîtrise des coûts, la flexibilité, la création d'un mécanisme d'apprentissage et l'innovation. 


\section{La maîtrise des coûts :}

Dans le cadre de l'entreprise réseau, la maîtrise des coûts est liée à la transformation des frais fixes en frais variables et la diminution du poids des investissements qui auraient dû être faits en l'absence de partenaires. En se désengageant des activités jugées les moins prioritaires, l'entreprise remplace les investissements et les immobilisations par la rémunération de prestataires externes, ce qui lui permet aussi d'éviter la gestion des tensions internes. Les conséquences seront ainsi une meilleure flexibilité, un abaissement du seuil de rentabilité, un effet de levier financier considérable et des besoins en capitaux nettement moins importants (Fréry, 2001). Le partage des risques induit va permettre d'élargir les économies d'échelle en augmentant la spécialisation des entreprises membres du réseau.

La réduction des coûts est également engendrée par la diminution spectaculaire des coûts de transaction. Ces derniers représentent les coûts de recherche du fournisseur qui offre une meilleure qualité des produits et des services ainsi que les coûts engendrés par la gestion de la relation. Cet avantage augmente avec une communication rapide et riche, basée sur la confiance, et qui rend les interfaces de production complémentaires et les interfaces informationnelles particulièrement flexibles et efficaces (Fréry, 2001). C'est ce qui explique la relation entre échange de l'information et la réduction des coûts.

Ce mode de fonctionnement en réseau et le désengagement des activités les moins maîtrisées va transformer les coûts indirects en coûts directs. Le fait de remplacer les calculs de coût complet et des clés de répartition parfois arbitraires par les montants payés aux intervenants accroît la pertinence des informations fournies par la comptabilité analytique et réduit les frais de structure, notamment en termes de contrôle de gestion. "Une des raisons principales qui pousse les responsables de petites entreprises à externaliser leur informatique ou leur comptabilité est la bien meilleure visibilité du coût de ces activités lorsqu'il suffit de rémunérer un prestataire » (Fréry, 2001).

\section{La flexibilité :}

Le fonctionnement dans le cadre d'une entreprise réseau permet aux différents membres de réagir rapidement aux aléas économiques (Julien et al., 2003). En effet, grâce à ce mode d'organisation, la communication devient rapide du fait que les organisations ont des relations partenariales. Les contrats partenariaux sont des contrats cadres à long terme (trois à cinq ans) partiellement explicites et habituellement suffisamment généraux pour faciliter toutes sortes d'ajustements en cours de route (Julien et al., 2003). Ceci permet d'améliorer la flexibilité à court terme.

À plus long terme, la division du travail entre les entreprises représente le principal facteur qui facilite le changement, l'intégration des innovations et des technologies développées grâce à l'échange entre les partenaires.

\section{La création d'un mécanisme d'apprentissage :}

L'apprentissage, générateur de diversité et garant d'une certaine cohérence, caractérise la capacité des acteurs du réseau à affronter la complexité et l'incertitude liées aux transformations de leur environnement et à modifier, au cours du temps, leurs comportements. L'entreprise n'a plus un comportement seulement réactif mais elle cherche à générer, par la remise en cause de routines de gestion et l'élaboration de nouvelles règles, de nouvelles formes techniques et sociales d'organisation destinées à améliorer la productivité. L'apprentissage représente ainsi un déterminant fondamental de l'avantage concurrentiel (Ketata et al., 2000) ${ }^{6}$.

Plusieurs recherches ont porté sur le concept d'apprentissage au sein des réseaux. Les interactions au sein d'un réseau mettent en présence des acteurs, des activités et des ressources. Chaque acteur, c'est-à-dire chaque entreprise membre du réseau peut être décrite d'après trois dimensions : les activités qu'elle accomplit, les ressources qu'elle contrôle ou possède, et la connaissance qu'elle a des activités, des ressources et des autres membres du réseau. Une activité correspond à la combinaison, au développement et à la création collective de ressources en utilisant les mêmes intrants.

Gérard Koenig (1994) ${ }^{7}$ définit l'apprentissage organisationnel comme un phénomène collectif d'acquisition et d'élaboration de connaissances 
qui, plus ou moins profondément, plus ou moins durablement, modifie la gestion des situations et les situations elles-mêmes. En ce qui concerne la diffusion et la circulation des nouvelles connaissances, l'impact de l'apprentissage croît avec le nombre et la diversité des interprétations produites. Partant du constat que le développement des relations représente un mode d'apprentissage, l'entreprise réseau pourrait être considérée comme une organisation apprenante (Fréry, 2001). La création d'un avantage concurrentiel apparaît alors comme le résultat d'un processus de construction de ressources spécifiques, et notamment cognitives.

Ce processus d'apprentissage nécessite l'accroissement de l'interdépendance entre les membres du réseau et le développement de synergies.

\section{L'innovation :}

L'innovation représente la raison d'être de l'entreprise réseau. Mais il devient de plus en plus évident que l'innovation provient d'informations riches et variées issues en grande partie de l'extérieur de l'entreprise. La nécessité de nouer des relations partenariales et de fonctionner en réseau s'explique par le fait que l'innovation comme l'information nouvelle la soutenant découlent nettement d'un processus collectif (Julien et al., 2003). En effet, le contrôle de l'information permet de développer des avantages concurrentiels au moyen d'innovations (Julien et al., 2003).
Ces dernières internalisées dans le réseau et associées à des liens de communication étroits accélèrent les échanges et le développement d'idées nouvelles pour l'innovation (Julien et al., 2003).

\section{La création d'un avantage concurrentiel apparaît alors comme le résultat d'un pro- cessus de construction de ressources spéci- fiques, et notamment cognitives.}

Dans le cadre du réseau, l'effort collectif doit permettre l'innovation et le changement, sinon le recours au marché devient plus intéressant. Elle consiste à améliorer les services pour mieux les adapter aux clients et à l'évolution de leur demande. La principale finalité de l'innovation est alors de créer de la valeur pour le client. L'entreprise peut intégrer l'innovation dans les procédés qui améliorent l'efficience avec laquelle les intrants sont transformés en produits. L'innovation peut aussi concerner les produits, pour qu'ils soient de meilleure qualité, moins chers et plus différenciés ainsi que par un lancement plus rapide des nouveaux produits. Pour Kaplan et Norton (2001) ${ }^{8}$, « il existe une relation directe entre la valeur d'une entreprise et son aptitude à innover, à améliorer et à apprendre. Ce n'est que dans la mesure où elle est capable de porter sur le marché des produits innovants, de créer encore plus de valeur pour le client et d'accroître l'efficacité des opérations qu'une entreprise peut envisager de pénétrer de nouveaux marchés et d'augmenter ses revenus et ses marges».

Tableau 1 : Les avantages de l'entreprise réseau

\begin{tabular}{|l|l|}
\hline Avantage & Implication \\
\hline Maîtrise des coûts & $\begin{array}{l}\text { Effet de levier financier, flexibilité, besoin en capitaux } \\
\text { moins important }\end{array}$ \\
\hline Flexibilité & $\begin{array}{l}\text { Adaptation aux attentes des clients et de l'ensemble des } \\
\text { parties prenantes }\end{array}$ \\
\hline Création d'un mécanisme d'apprentissage & $\begin{array}{l}\text { Développement de compétences, développement de res- } \\
\text { sources cognitives }\end{array}$ \\
\hline Innovation & $\begin{array}{l}\text { Meilleure satisfaction des clients, intégration des normes } \\
\text { écologiques et environnementales }\end{array}$ \\
\hline
\end{tabular}

Le fonctionnement dans le cadre d'une entreprise réseau représente une solution pour faire face aux exigences imposées par l'environnement. En effet, l'entreprise réseau représente une source de création de valeur et davantage concurrentielle non pas en tant que telle mais à travers les processus qu'elle 
sécrète. Ces processus trouvent leurs sources dans le recentrage sur le corps de métier et la coopération dense autour d'une dimension verticale de la production et consistent en une dynamique d'échange d'informations, d'apprentissage et d'innovations conjuguées.

Le contrôle dans le cadre de l'entreprise réseau est effectué par l'entreprise pivot.

\section{Les enjeux de l'entreprise réseau}

Malgré les avantages reconnus de l'entreprise réseau, il existe certains enjeux qui sont liés à ce mode d'organisation. Ces enjeux varient selon les réseaux et leur environnement réel, et peuvent différer selon que l'on adopte le point de vue de la firme pivot ou des partenaires. Ces enjeux sont principalement liés à la difficulté de contrôle et la perte de pouvoir ainsi que le risque d'apparition de comportements opportunistes.

\section{La difficulté de contrôle et la perte de pouvoir :}

Ce problème est lié à la difficulté de contrôle et d'intégration d'une chaîne de valeur que l'on ne possède pas, et notamment à la crainte de voir des ressources clés et des compétences fondamentales échapper à la maîtrise directe des décideurs stratégiques. Ainsi une perte de pouvoir sera constatée tant pour les partenaires que pour l'entreprise pivot. Cette dernière, généralement de grande taille, ne peut plus fonctionner comme si elle était seule face à la concurrence. Elle doit tenir compte de ses partenaires, perdant ainsi le contrôle traditionnel que l'on trouve généralement dans les grandes entreprises intégrées verticalement. Cela suppose des compromis importants pas toujours évidents, notamment dans les processus traditionnels de gestion.

Le contrôle dans le cadre de l'entreprise réseau est effectué par l'entreprise pivot. Les auteurs qui ont étudié les relations dans les réseaux d'entreprises s'entendent sur la nécessité d'un noyau, appelé aussi hub firm ou firme locale, firme pivot, broker, strategic center, agence stratégique, ou encore server, distributor ou integrator. D'une manière générale, il apparaît qu'en l'absence d'un organe formel de gouvernance, une structure interentreprises perd une grande partie de ses avantages, pour devenir un simple marché, avec toutes les déficiences que les économistes associent à ce mode de régulation.

Pour Fréry, le rôle du noyau s'articule autour de trois $\mathrm{C}$ formés par le triptyque Conception, Coordination et Contrôle. Le noyau conçoit la chaîne de valeur en repérant les opportunités de développement de nouvelles activités, à la manière d'une cellule d'analyse stratégique au sein d'un groupe, puis en contactant et sélectionnant les intervenants extérieurs susceptibles d'y participer. Il coordonne ensuite la structure obtenue en assurant la gouvernance des transactions entre les partenaires, que ce soit par la gestion de flux physiques (intermédiation par achat de la production des maillons amonts et revente aux maillons avals), ou par la maîtrise des flux d'information (constitution d'un réseau EDI ${ }^{9}$, récolte et redistribution de données). Enfin, le noyau contrôle la structure en s'assurant du niveau de qualité atteint par les intervenants, en détenant des actifs stratégiques indispensables à la survie de l'ensemble (marque, réseau logistique, technologie spécifique, interface privilégiée avec le marché ou les prescripteurs, etc.), ou en dépêchant régulièrement des auditeurs chez les sous-traitants.

\section{Le risque d'apparition de comportements oppor- tunistes :}

Dans la gestion des relations interentreprises, des comportements opportunistes entre les partenaires peuvent être détectés (Lakhal et al., 1999 ; Julien et al., 2003). Un comportement opportuniste est défini comme étant des actions entreprises par l'un des partenaires d'une relation afin de profiter d'une situation sans tenir compte de l'intérêt de l'autre. Il s'agit de favoriser un gain à court terme par rapport à une relation durable et adopter un comportement opportuniste dans le cadre de la relation.

Ce comportement opportuniste semble être associé à trois types de problèmes : des problèmes informationnels, des problèmes de valeurs relationnelles et des problèmes de valeur du marché (Lakhal et al., 1999).

Les problèmes informationnels sont générés par une capacité d'information asymétrique de la part des partenaires en présence. Ces derniers n'ont pas le même potentiel, ni la même capacité de collecte et d'analyse des informations et des données économiques relatives aux enjeux du domaine contractuel. 
Le partenaire ayant le plus de pouvoir pourrait tenter de profiter de la situation étant donné qu'il disposerait de certaines informations que son partenaire n'a pas.

S'agissant des problèmes de valeurs relationnelles, ils apparaissent lorsque la relation commence à générer des bénéfices. La valeur accordée au montant de ces bénéfices peut être différente. Chaque partenaire essaie de comparer sa part des bénéfices à l'effort fourni dans le cadre de la relation. Si un des partenaires arrive à la conclusion que le bénéfice reçu ne récompense pas son effort, il risque d'avoir un comportement opportuniste afin de compenser ce qu'il juge être une inégalité.

Enfin, les problèmes de valeur du marché désignent la divergence entre le prix convenu dans le contrat et le prix réel du marché. Suite à un pareil constat un des partenaires peut essayer de livrer, par exemple, des produits de moindre qualité ou ne pas respecter les délais de livraison.

Pour réduire le risque d'apparition de comportements opportunistes, il existe deux solutions privilégiées. Premièrement, les partenaires devraient favoriser les relations à long terme par rapport aux relations à court terme, afin de rentabiliser les investissements spécifiques effectués dans le cadre de leur relation. Deuxièmement, il faut aussi avantager les relations à plusieurs liens (par exemple, des liaisons sur le plan commercial avec des liaisons en production, soutenues par des liaisons en recherche et développement).

Mieux gérer ces enjeux dépend ainsi du comportement des entreprises membres du réseau ainsi que du degré de dépendance entre ces derniers. En effet, l'interdépendance de l'entreprise avec ses partenaires présente plusieurs défis à relever et une diversité de problèmes à gérer liés à la dimension sociale ou relationnelle. La dépendance se base sur trois éléments : l'importance des ressources, la discrétion concernant l'extension de ces ressources et la rareté des alternatives possibles pour les parties. Lakhal et al. (1999) proposent cinq facteurs de dépendance :

«1- l'importance de la relation pour la partie concernée (on est plus dépendant si la relation est jugée importante) ;
2- la performance de la relation (la dépendance augmente si les revenus qu'on tire d'une relation sont élevés par rapport aux autres alternatives);

3- la concentration des alternatives (la dépendance augmente si le nombre d'alternatives est très restreint) ;

4- le potentiel de développement des alternatives (le lien est plus grand si le potentiel de développement des alternatives est faible);

5- les investissements spécifiques effectués par une entreprise dans le cadre d'une relation interentreprises augmentent sa dépendance. »

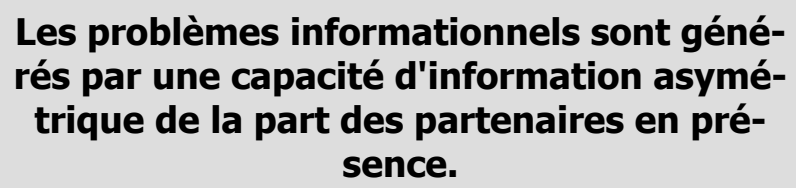

Plus les liens de coopération et de dépendance seront importants, plus la relation sera meilleure. Les membres du réseau pourront profiter au mieux des potentialités offertes par ce mode d'organisation interentreprises.

\section{Conclusion}

Le développement du modèle de l'entreprise réseau est principalement lié aux exigences et aux contraintes environnementales qui s'exercent sur l'entreprise. Cette forme de coopération interentreprises s'inscrit dans le cadre de la recherche de nouvelles modalités d'organisation et, en particulier, de coopération. Les entreprises orientent leurs efforts vers le redéploiement de leurs stratégies dans un objectif de création de valeur. Elles effectuent un processus de recentrage sur les métiers principaux que l'on peut assimiler à une spécialisation de la part de chaque entreprise sur les secteurs d'activités qu'elle peut contrôler et dont elle possède les ressources et maîtrise les compétences. Elles externalisent, par contre, des activités à d'autres organisations capables de les réaliser à moindre coût. L'entreprise est ainsi considérée comme un portefeuille de ressources et de compétences. En effet, l'entreprise était considérée pendant les trente glorieuses comme un portefeuille de produits, pendant les années 80 comme un portefeuille d'activités, pendant les années 90 comme un portefeuille de compétences et, enfin, pendant les années 2000 
comme un portefeuille d'alliances aux frontières floues.

Cependant, l'entreprise réseau peut être confrontée à certains enjeux pouvant entraver son succès. Malgré ces enjeux, l'entreprise réseau présente des qualités théoriques qui permettent de tirer un meilleur parti de la démarche ressources et compétences. Elle permet de bénéficier d'un remarquable effet de levier sur les ressources des partenaires, mais aussi de leurs compétences. Elle favorise également la maîtrise des coûts, le gain de flexibilité, l'accès à des sources plus variées et à une expertise de pointe, la réduction du risque associé aux projets et à l'allègement de la structure interne.

\section{Notes et références}

1 Julien, P.-A., Raymond L., Jacob R. et Abdul-Nour G. (2003). L'entreprise-réseau. Presses Universitaires du Québec.

2 Paché, G. et Paraponaris C. (1993, 1994, 2006). L'entreprise en réseau. Paris. PUF.

3 Gianfaldoni, P., Guilhon, B. et P. Trinquet (1997). La firme-réseau dans le BTP, Plan Construction et Architecture, Paris.
4 Fréry, F. (2001). «Entreprises virtuelles et réalités stratégiques. » Revue Française de Gestion n¹33 (Mars-Avril-Mai).

5 Paché, G. et Bacus-Montfort I. (2003). « Le management logistique intégré». Problèmes économiques. 15 Janvier $2003 n^{\circ} 2.792$

6 Ketata N., Poulin D., Kettani O. (2000). « Les déterminants de l'avantage concurrentiel de l'entreprise réseau ». Document de travail 2000-025. Centre de recherche sur les technologies de l'organisation réseau. Édition électronique de l'Université Laval. Http ://www.fsa.ulaval.ca/rd

7 Koenig Gérard. (1994). «L’apprentissage organisationnel : repérage des lieux ». Revue Française de Gestion. Janvier- Février. p78.

8 Kaplan R. et Norton D. (2001). In Les systèmes de mesure de la performance. Éditions d'organisation.

9 Electronic Data Interchange (Échange de Données Informatisées) : est un outil au service de l'échange électronique consistant à transporter automatiquement de l'application informatique d'une entreprise à l'application informatique d'une autre entreprise, par des moyens de télécommunication, des données structurées selon une normalisation convenue à l'avance. 


\section{Publicité}

«Min. Affaires municipales et Régions » 